08

\title{
Спектроскопия иттриевого скандата, легированного ионами тулия
}

\author{
() Е.А. Добрецова, О.К. Алимов, С.Я. Русанов, В.В. Кашин, В.В. Воронов, Д.А. Гурьев, \\ С.А. Кутовой, В.И. Власов, В.Б. Цветков
}

Институт общей фризики имени А.М. Прохорова РАН,

Москва, Россия

E-mail: elenadobretsova89@gmail.com

Поступила в Редакцию 8 июля 2021 г.

В окончательной редакции 13 июля 2021 г.

Принята к публикации 16 июля 2021 г.

\begin{abstract}
Методом минипьедестала с лазерным нагревом выращено кристаллическое волокно иттриевого скандата, легированного ионами тулия. Полученный кристалл относится к структурному типу биксбиита, имеет кубическую структуру и кристаллизуется в пространственной группе Ia $\overline{3}$. Методом рентгенофазового анализа найдены параметры элементарной ячейки $a=10.224(1) \AA$. Спектрально-кинетические исследования кристаллического волокна позволили обнаружить два независимых по симметрии оптических центра ионов $\mathrm{Tm}^{3+}$.
\end{abstract}

Ключевые слова: биксбиит, оптический центр, редкоземельный ион, метод мини-пьедестала с лазерным нагревом.

DOI: 10.21883/FTT.2021.12.51657.22s

\section{1. Введение}

Оксиды скандия и иттрия со структурным типом биксбиита характеризуются высокой теплопроводностью (в два раза больше, чем у иттрий-алюминиевого граната $($ ИАГ) $)$ [1] и широким окном прозрачности от 0.25 до $9.6 \mu \mathrm{m}$ [2]. Такие свойства особенно ценятся при создании твердотельных лазеров [3-7], поэтому $\mathrm{Y}_{2} \mathrm{O}_{3}$ и $\mathrm{Sc}_{2} \mathrm{O}_{3}$, легированные редкоземельными ионами, могут стать достойной альтернативой кристаллам ИАГ. Оксидные материалы считаются перспективными для различных применений, среди которых: твердотельные светоизлучательные устройства $[8,9]$, высокоэффективные люминесцентные материалы $[10,11]$, редкоземельные магниты $[12,13]$, материалы для магнитооптической записи [14] и т.д.

Иттриевый скандат - полиморфное соединение. При этом его высокотемпературная модификация, так же как оксиды иттрия [15] и скандия [16], принадлежит к структурному типу биксбиита с пространственной группой $\operatorname{Ia} \overline{3}$ [17]. В кристаллической структуре выделяют одну позицию кислорода с локальной симметрией $C_{1}$ (позиция Вайкоффа 48e) и две независимые по симметрии позиции катионов: с локальной симметрией $C_{3 i}$ (позиция Вайкоффа $8 b$ ) и локальной симметрией $C_{2}$ (позиция Вайкоффа 24d) (рис. 1). Ранее проведенные исследования легированного $\mathrm{Nd}^{3+}$ кристалла $\mathrm{YScO}_{3}$ со структурным типом биксбиита показали, что $\mathrm{Y}^{3+}$ и $\mathrm{Sc}^{3+}$ статистически распределены между двумя позициями в соотношении примерно 1:1 в каждой [18]. При этом $\mathrm{Nd}^{3+}$ свободно замещает основные катионы. Более того, большие октаэдрические пустоты, образующиеся между основными структурными полиэдрами, теоретически могут служить в качестве дополнительных позиций для примесных редкоземельных ионов. Чтобы изучить влияние величины радиуса легирующего иона на структурные и спектроскопические характеристики $\mathrm{YScO}_{3}$, в настоящей работе были синтезированы и охарактеризованы кристаллические волокна иттриевого скандата, легированного $\mathrm{Tm}^{3+}$.

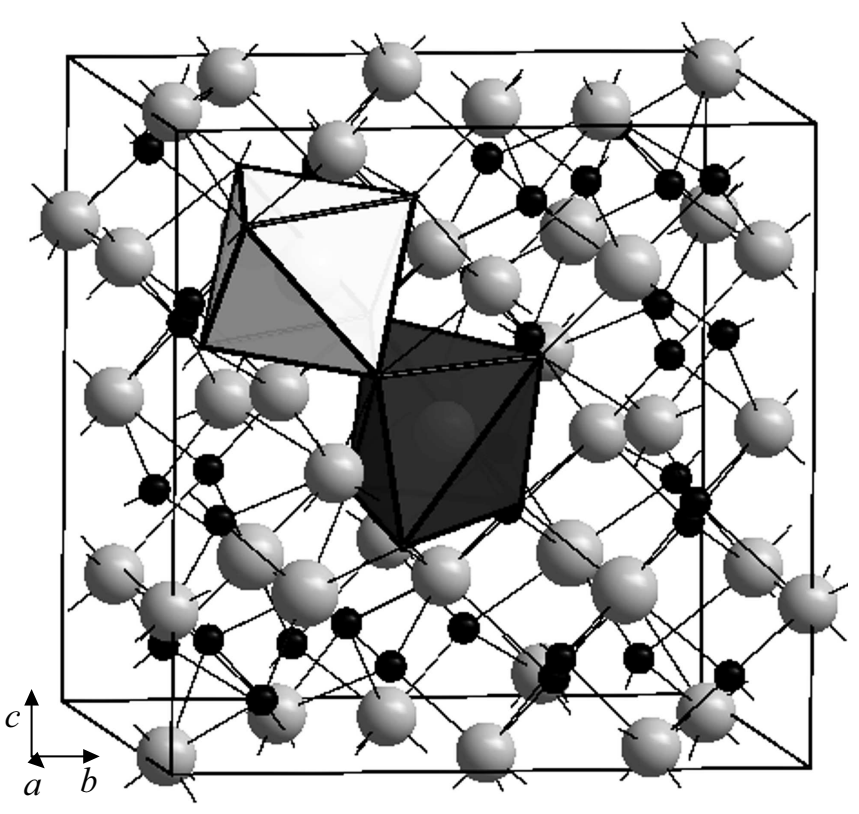

Рис. 1. Кристаллическая структура иттриевого скандата с пространственной группой $I a \overline{3}$. Серым обозначены ионы $\mathrm{Y}^{3+}$ и $\mathrm{Sc}^{3+}$, статистически распределенные между двумя независимыми по симметрии позициями; черным - ионы кислорода, белым и темно-серым - координационные полиэдры с локальной симметрией $C_{3 i}$ и $C_{2}$ соответственно. 


\section{2. Экспериментальная часть}

Для выращивания кристалла $\mathrm{Tm}^{3+}: \mathrm{YScO}_{3}$ применялся метод минипьедестала с лазерным нагревом $[19,20]$. Данная техника позволяет синтезировать кристаллы в форме волокна и хорошо подходит для получения тугоплавких материалов и соединений с разупорядоченной кристаллической структурой, поскольку сопровождается высокотемпературным нагревом и последующим быстрым охлаждением.

Часть полученного кристаллического волокна была перетерта в агатовой ступке и охарактеризована с помощью рентгенофазового анализа. Запись дифрактограммы проводилась на рентгеновском дифрактометре Bruker D8 Discover A25 DaVinsi Design с интервалом сканирования $2 \theta=15-80^{\circ}$, шагом сканирования $0.02^{\circ}$, экспозицией в шаге $1.0 \mathrm{~s}$. В качестве источника излучения использовали керамическую рентгеновскую трубку Siemens KFL с излучением $\mathrm{Cu}_{\alpha}(U=40 \mathrm{kV}$, $I=40 \mathrm{~mA}, \lambda=0.154 \mathrm{~nm})$. Спектр был обработан с помощью программы EVA версия 2.1 и расшифрован с использованием базы данных PDF-2 версии 2011 года. Индицирование спектров и определение параметров элементарной ячейки проводилось с помощью программы TOPAS версия 4.2.

Спектры комбинационного рассеяния были зарегистрированы с помощью рамановского портативного экспресс-анализатора EnSpectr $R 532$ (длина волны лазерного излучения $532 \mathrm{~nm}$ ), оснащенного микроскопом Olympus. Измерения проводились при сорокократном увеличении, фокусировка производилась примерно в сердцевину волокна. Каждый спектр представляет собой разность между спектром, снятым при работающем лазере, и темновым спектром. Вычитание одного спектра из другого осуществлялось программой EnSpectr Pro автоматически.

Спектрально-кинетические характеристики кристаллического волокна $\mathrm{Tm}^{3+}: \mathrm{YScO}_{3}$ были получены с использованием методов селективной лазерной спектроскопии. Спектры люминесценции зарегистрированы на монохроматоре МДР-23. В качестве фотоприемников использовались фотоумножители Hamamatsu $R 5108$ и $\mathrm{PbS}$ детектор Thorlabs PDA30G-EC с усилением. В качестве источника возбуждения использовали перестраиваемый лазерный комплекс LQ529B-LP 604 (Соларис ЛC).

\section{3. Результаты и обсуждение}

Для выращивания кристаллов высокочистые порошки (> 99.999\%) оксидов скандия, иттрия и тулия $(0.1 \mathrm{~mol} . \%)$ были смешаны и перетерты в агатовой ступке до гомогенного состояния. Затем смесь поместили в платиновый тигель и прокаливали при температуре $1100^{\circ} \mathrm{C}$ в течение $48 \mathrm{~h}$. Под давлением $20 \mathrm{MPa}$ был получен стержень, соответствующий составу порошковой смеси $0.1 \mathrm{~mol} . \% \mathrm{Tm}_{2} \mathrm{O}_{3}-\mathrm{Y}_{2} \mathrm{O}_{3}-\mathrm{Sc}_{2} \mathrm{O}_{3}$, длиной около $50 \mathrm{~mm}$ и диаметром $1 \mathrm{~mm}$.

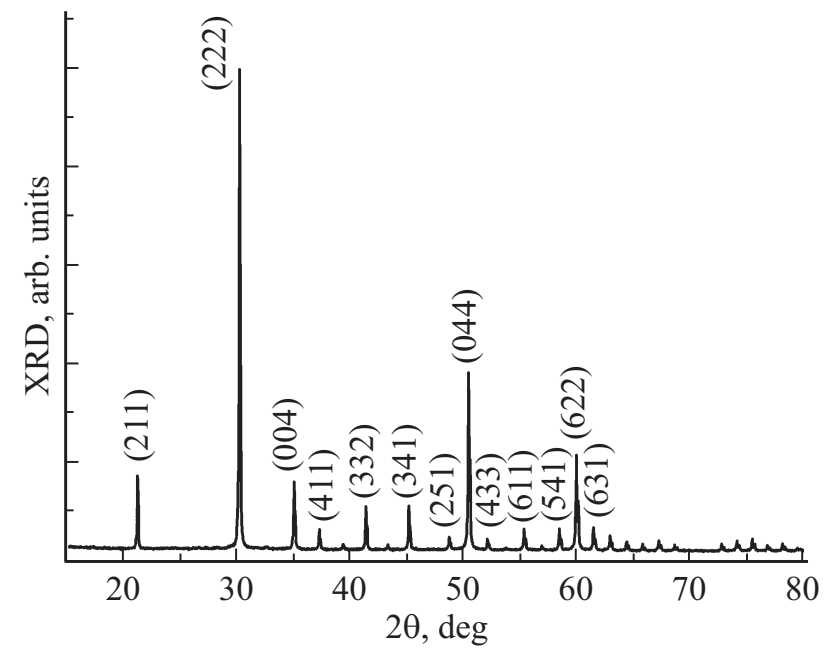

Рис. 2. Порошковая рентгенограмма $\mathrm{Tm}^{3+}: \mathrm{YScO}_{3}$. Наиболее интенсивные пики проиндексированы.

Торец заранее приготовленной цилиндрической заготовки (стержень состава $0.1 \mathrm{~mol} . \% \mathrm{Tm}_{2} \mathrm{O}_{3}-\mathrm{Y}_{2} \mathrm{O}_{3}-\mathrm{Sc}_{2} \mathrm{O}_{3}$ ), расположенной вертикально, нагревался под действием излучения $\mathrm{CO}_{2}$ лазера. В результате этого на пьедестале (торце заготовки) образовывалась капля расплава. В данную область был подведен затравочный кристалл. В качестве затравки использовали диоксид циркония, стабилизированный оксидом иттрия. При соприкосновении затравки с расплавом и последующем вытягивании кристалла со скоростью $80 \mathrm{~mm} / \mathrm{h}$, на конце затравки образовалось кристаллическое волокно в форме, близкой к цилиндрической. Одновременно с вытягиванием растущего кристалла, в зону нагрева подавалась заготовка для компенсации расхода материала, уносимого из зоны расплава выращенным волокном. В результате синтеза было получено кристаллическое волокно диаметром $0.6 \mathrm{~mm}$ и длиной $50 \mathrm{~mm}$.

Структурные характеристики кристаллического волокна, часть которого была перетерта в порошок, были получены методом рентгенофазового анализа. Рентгенограмма $\mathrm{Tm}^{3+}: \mathrm{YScO}_{3}$ содержит хорошо выраженные узкие пики (рис. 2), что указывает на высокую степень кристалличности. Согласно рентгенодифракционным данным, соединение $\mathrm{Tm}^{3+}: \mathrm{YScO}_{3}$ гомогенно и принадлежит к структурному типу биксбиита (пространственная группа $I a \overline{3})$, параметры элементарной ячейки определены как $a=10.224(1) \AA$. Индексация пиков приведена в табл. 1. [18]. Наиболее интенсивные пики обозначены на рис. 2.

Спектры комбинационного рассеяния были получены для исходных материалов (порошки $\mathrm{Y}_{2} \mathrm{O}_{3}$ и $\mathrm{Sc}_{2} \mathrm{O}_{3}$ ) и кристаллического волокна $\mathrm{Tm}^{3+}: \mathrm{YScO}_{3}$ (рис. 3).

Примитивная ячейка иттриевого скандата содержит 40 атомов (8 формульных единиц). Таким образом, теоретико-групповой анализ приводит нас к следующему распределению 120 фононных мод в $\Gamma=0$ точке зоны 
Таблица 1. Индексация пиков рентгенограммы $\mathrm{Tm}^{3+}: \mathrm{YScO}_{3}$. $h k l$ - индексы отражения, $d-$ межплоскостное расстояние, $2 \theta$ - угол дифракции, $I-$ интенсивность пиков отражений, $m$ - кратность отражений.

\begin{tabular}{|c|c|c|c|c|c|c|c|c|c|}
\hline № & $h$ & $k$ & $l$ & $m$ & $d$ & $d[18]$ & $2 \theta$ & $2 \theta[18]$ & $I[18]$ \\
\hline 1 & 0 & 0 & 2 & 6 & & 5.1083 & & 17.346 & 0.36 \\
\hline 2 & 2 & 1 & 1 & 24 & 4.1756 & 4.1709 & 21.261 & 21.285 & 13.54 \\
\hline 3 & 0 & 2 & 2 & 12 & 3.6162 & 3.6121 & 24.598 & 24.626 & 0.04 \\
\hline 4 & 2 & 2 & 2 & 8 & 2.9526 & 2.9493 & 30.246 & 30.28 & 100.00 \\
\hline 5 & 3 & 2 & 1 & 24 & 2.7336 & 2.7305 & 32.734 & 32.772 & 0.07 \\
\hline 6 & 2 & 3 & 1 & 24 & 2.7336 & 2.7305 & 32.734 & 32.772 & 0.03 \\
\hline 7 & 0 & 0 & 4 & 6 & 2.5570 & 2.5542 & 35.065 & 35.106 & 18.45 \\
\hline 8 & 4 & 1 & 1 & 24 & 2.4108 & 2.4081 & 37.268 & 37.312 & 4.62 \\
\hline 9 & 0 & 4 & 2 & 12 & 2.2871 & 2.2845 & 39.365 & 39.411 & 0.48 \\
\hline 10 & 4 & 0 & 2 & 12 & 2.2871 & 2.2845 & 39.365 & 39.411 & 0.73 \\
\hline 11 & 3 & 3 & 2 & 24 & 2.1806 & 2.1782 & 41.372 & 41.421 & 8.36 \\
\hline 12 & 4 & 2 & 2 & 24 & 2.0878 & 2.0855 & 43.302 & 43.353 & 1.03 \\
\hline 13 & 3 & 4 & 1 & 24 & 2.0059 & 2.0036 & 45.166 & 45.219 & 5.15 \\
\hline 14 & 4 & 3 & 1 & 24 & 2.0059 & 2.0036 & 45.166 & 45.219 & 3.96 \\
\hline 15 & 2 & 5 & 1 & 24 & 1.8674 & 1.8653 & 48.724 & 48.782 & 1.79 \\
\hline 16 & 5 & 2 & 1 & 24 & 1.8674 & 1.8653 & 48.724 & 48.782 & 1.48 \\
\hline 17 & 0 & 4 & 4 & 12 & 1.8081 & 1.8061 & 50.432 & 50.492 & 47.56 \\
\hline 18 & 4 & 3 & 3 & 24 & 1.7541 & 1.7521 & 52.098 & 52.161 & 2.14 \\
\hline 19 & 0 & 0 & 6 & 6 & 1.7047 & 1.7028 & 53.728 & 53.793 & 0.40 \\
\hline 20 & 4 & 4 & 2 & 24 & 1.7047 & 1.7028 & 53.728 & 53.793 & 0.07 \\
\hline 21 & 6 & 1 & 1 & 24 & 1.6592 & 1.6574 & 55.324 & 55.392 & 3.47 \\
\hline 22 & 3 & 5 & 2 & 24 & 1.6592 & 1.6574 & 55.324 & 55.392 & 0.96 \\
\hline 23 & 5 & 3 & 2 & 24 & 1.6592 & 1.6574 & 55.324 & 55.392 & 1.15 \\
\hline 24 & 0 & 6 & 2 & 12 & 1.6172 & 1.6154 & 56.890 & 56.96 & 0.78 \\
\hline 25 & 6 & 0 & 2 & 12 & 1.6172 & 1.6154 & 56.890 & 56.96 & 0.42 \\
\hline 26 & 5 & 4 & 1 & 24 & 1.5782 & 1.5765 & 58.429 & 58.501 & 2.68 \\
\hline 27 & 4 & 5 & 1 & 24 & 1.5782 & 1.5765 & 58.429 & 58.501 & 1.86 \\
\hline 28 & 6 & 2 & 2 & 24 & 1.5419 & 1.5402 & 59.942 & 60.017 & 27.23 \\
\hline 29 & 6 & 3 & 1 & 24 & 1.5081 & 1.5064 & 61.433 & 61.51 & 3.43 \\
\hline 30 & 3 & 6 & 1 & 24 & 1.5081 & 1.5064 & 61.433 & 61.51 & 3.13 \\
\hline 31 & 4 & 4 & 4 & 8 & 1.4763 & 1.4746 & 62.903 & 62.982 & 3.66 \\
\hline 32 & 4 & 5 & 3 & 24 & 1.4465 & 1.4448 & 64.354 & 64.435 & 0.60 \\
\hline 33 & 5 & 4 & 3 & 24 & 1.4465 & 1.4448 & 64.354 & 64.435 & 1.27 \\
\hline 34 & 0 & 6 & 4 & 12 & 1.4184 & 1.4168 & 65.788 & 65.871 & 1.13 \\
\hline 35 & 6 & 0 & 4 & 12 & 1.4184 & 1.4168 & 65.788 & 65.871 & 0.22 \\
\hline 36 & 7 & 2 & 1 & 24 & 1.3919 & 1.3903 & 67.205 & 67.291 & 1.09 \\
\hline 37 & 2 & 7 & 1 & 24 & 1.3919 & 1.3903 & 67.205 & 67.291 & 1.99 \\
\hline 38 & 6 & 3 & 3 & 24 & 1.3919 & 1.3903 & 67.205 & 67.291 & 0.77 \\
\hline 39 & 5 & 5 & 2 & 24 & 1.3919 & 1.3903 & 67.205 & 67.291 & 0.01 \\
\hline 40 & 6 & 4 & 2 & 24 & 1.3668 & 1.3653 & 68.608 & 68.696 & 0.23 \\
\hline 41 & 4 & 6 & 2 & 24 & 1.3668 & 1.3653 & 68.608 & 68.696 & 1.28 \\
\hline 42 & 7 & 3 & 2 & 24 & 1.2990 & 1.2975 & 72.741 & 72.836 & 0.08 \\
\hline 43 & 5 & 6 & 1 & 24 & 1.2990 & 1.2975 & 72.741 & 72.836 & 0.00 \\
\hline 44 & 3 & 7 & 2 & 24 & 1.2990 & 1.2975 & 72.741 & 72.836 & 0.97 \\
\hline 45 & 6 & 5 & 1 & 24 & 1.2990 & 1.2975 & 72.741 & 72.836 & 0.80 \\
\hline 46 & 0 & 0 & 8 & 6 & 1.2785 & 1.2771 & 74.098 & 74.195 & 4.70 \\
\hline 47 & 8 & 1 & 1 & 24 & 1.2590 & 1.2576 & 75.445 & 75.545 & 1.74 \\
\hline 48 & 4 & 7 & 1 & 24 & 1.2590 & 1.2576 & 75.445 & 75.545 & 0.76 \\
\hline 49 & 7 & 4 & 1 & 24 & 1.2590 & 1.2576 & 75.445 & 75.545 & 0.58 \\
\hline 50 & 5 & 5 & 4 & 24 & 1.2590 & 1.2576 & 75.445 & 75.545 & 0.54 \\
\hline 51 & 0 & 8 & 2 & 12 & 1.2403 & 1.2389 & 76.784 & 76.886 & 0.54 \\
\hline 52 & 8 & 0 & 2 & 12 & 1.2403 & 1.2389 & 76.784 & 76.886 & 0.92 \\
\hline 53 & 6 & 4 & 4 & 24 & 1.2403 & 1.2389 & 76.784 & 76.886 & 0.46 \\
\hline 54 & 6 & 5 & 3 & 24 & 1.2225 & 1.2211 & 78.116 & 78.221 & 1.04 \\
\hline 55 & 5 & 6 & 3 & 24 & 1.2225 & 1.2211 & 78.116 & 78.221 & 1.07 \\
\hline 56 & 0 & 6 & 6 & 12 & 1.2054 & 1.204 & 79.441 & 79.548 & 1.34 \\
\hline 57 & 8 & 2 & 2 & 24 & 1.2054 & 1.204 & 79.441 & 79.548 & 0.03 \\
\hline
\end{tabular}

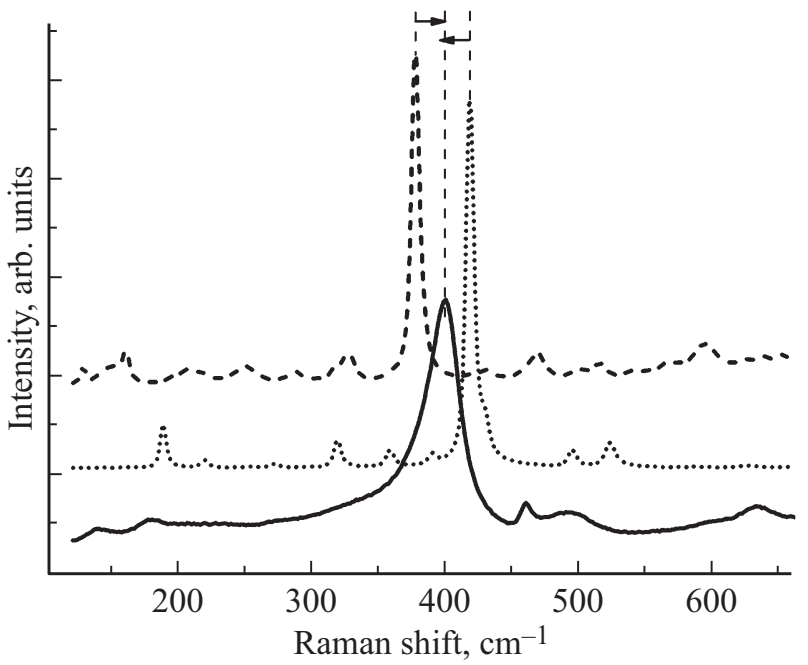

Рис. 3. Спектры комбинационного рассеяния $\mathrm{Y}_{2} \mathrm{O}_{3}$ (штриховая линия) и $\mathrm{Sc}_{2} \mathrm{O}_{3}$ (пунктирная линия) и кристаллического волокна $\mathrm{Tm}^{3+}: \mathrm{YScO}_{3}$ (сплошная линия).

Бриллюэна [21,22]:

$$
\begin{aligned}
\Gamma=\left(3 A_{g}+3 A_{u}+3 E_{g}+\right. & \left.3 e_{u}+9 F_{g}+9 F_{u}\right) \\
& \left(\text { локальная симметрия } C_{1}\right) \\
+\left(A_{g}+A_{u}+E_{g}+\right. & \left.E_{u}+5 F_{g}+5 F_{u}\right) \\
& \left(\text { локальная симметрия } C_{2}\right) \\
+\left(A_{u}+E_{u}+3 F_{u}\right) & \left(\text { локальная симметрия } C_{3 i}\left(S_{6}\right)\right) .
\end{aligned}
$$

После вычитания $1 F_{u}$ акустических мод остается $16 F_{u}$ ИК-активных, $\left(A_{g}+4 E_{g}+14 F_{g}\right)$ КРС-активных и $\left(5 A_{u}+5 E_{u}\right)$ оптически неактивных фононных мод.

В ряду $\mathrm{Sc}_{2} \mathrm{O}_{3} \rightarrow \mathrm{YScO}_{3} \rightarrow \mathrm{Y}_{2} \mathrm{O}_{3}$ наблюдается ,эффект масс“, при котором колебательные моды смещаются в более низкочастотную область при увеличении массы катиона. Спектральные линии иттриевого скандата уширены, вероятно, из-за присутствия обоих ионов $\mathrm{Y}^{3+}$ и $\mathrm{Sc}^{3+}$, статистически распределенных между двумя позициями в кристаллической структуре биксбиита. Из-за частичного перекрывания число линий в экспериментальном спектре кристалла скандата гораздо ниже числа линий, предсказанных теоретико-групповым анализом. В табл. 2 приведена идентификация КР-активных колебаний [23].

Спектры возбуждения люминесценции $\mathrm{Tm}^{3+}$ на переходе ${ }^{3} H_{6} \rightarrow{ }^{3} H_{5}$ при селективной регистрации спектров на $1945 \mathrm{~nm}$ (переход ${ }^{3} F_{4} \rightarrow{ }^{3} H_{6}$ ) были получены при температуре $77 \mathrm{~K}$ (рис. 4). В спектрах наблюдаются два наиболее интенсивных пика на 1202.4 и $1209.4 \mathrm{~nm}$. Соотношение интенсивностей этих спектральных линий меняется в зависимости от временной задержки регистрации относительно момента лазерного возбуждения, что позволяет говорить о присутствии в кристалле $\mathrm{YScO}_{3}$ двух типов оптических центров тулия с различными временами жизни. Известно [24], что электродипольные переходы запрещены для редкоземельных 
Таблица 2. КРС-активные колебания в $\mathrm{Y}_{2} \mathrm{O}_{3}, \mathrm{Sc}_{2} \mathrm{O}_{3}$ и $\mathrm{Tm}^{3+}$ : $\mathrm{YScO}_{3}$; ехр. - экспериментальные, calc. - расчетные

\begin{tabular}{|c|c|c|c|c|c|c|c|}
\hline Assignments [23] & $\mathrm{Sc}_{2} \mathrm{O}_{3}$, exp. [23] & $\mathrm{Sc}_{2} \mathrm{O}_{3}$, calc. $[23]$ & $\mathrm{Sc}_{2} \mathrm{O}_{3}$ & $\mathrm{YScO}_{3}$ & $\mathrm{Y}_{2} \mathrm{O}_{3}$, exp. [23] & $\mathrm{Y}_{2} \mathrm{O}_{3}$, calc. $[23]$ & $\mathrm{Y}_{2} \mathrm{O}_{3}$ \\
\hline $\mathrm{Ag}$ & $\begin{array}{l}221 \\
391 \\
495 \\
623\end{array}$ & $\begin{array}{l}220 \\
351 \\
498 \\
593\end{array}$ & $\begin{array}{c}220 \\
358 \\
494 \\
-\end{array}$ & $\begin{array}{c}180 \\
- \\
- \\
590\end{array}$ & $\begin{array}{c}162 \\
- \\
431 \\
-\end{array}$ & $\begin{array}{l}162 \\
306 \\
460 \\
553\end{array}$ & $\begin{array}{l}161 \\
318 \\
467 \\
542\end{array}$ \\
\hline$E_{g}$ & $\begin{array}{l}273 \\
359 \\
430 \\
626\end{array}$ & $\begin{array}{l}273 \\
332 \\
416 \\
590\end{array}$ & $\begin{array}{c}273 \\
- \\
429 \\
-\end{array}$ & $\begin{array}{c}- \\
- \\
400 \\
-\end{array}$ & $\begin{array}{c}194 \\
325 \\
- \\
-\end{array}$ & $\begin{array}{l}198 \\
290 \\
366 \\
555\end{array}$ & $\begin{array}{l}194 \\
288 \\
358 \\
567\end{array}$ \\
\hline$F_{g}$ & $\begin{array}{c}189 \\
202 \\
252 \\
319 \\
329 \\
359 \\
- \\
391 \\
419 \\
- \\
- \\
523 \\
587 \\
669\end{array}$ & $\begin{array}{l}192 \\
199 \\
250 \\
306 \\
311 \\
341 \\
368 \\
386 \\
412 \\
472 \\
491 \\
536 \\
605 \\
646\end{array}$ & $\begin{array}{c}189 \\
- \\
253 \\
- \\
319 \\
358 \\
- \\
390 \\
418 \\
- \\
494 \\
522 \\
624 \\
670\end{array}$ & $\begin{array}{c}140 \\
180 \\
- \\
- \\
- \\
- \\
- \\
- \\
400 \\
460 \\
- \\
495 \\
590 \\
630\end{array}$ & $\begin{array}{c}129 \\
138 \\
182 \\
235 \\
- \\
325 \\
- \\
- \\
377 \\
- \\
- \\
469 \\
- \\
592\end{array}$ & $\begin{array}{l}132 \\
138 \\
181 \\
234 \\
244 \\
288 \\
299 \\
338 \\
368 \\
416 \\
442 \\
495 \\
545 \\
581\end{array}$ & $\begin{array}{c}129 \\
152 \\
207 \\
217 \\
250 \\
288 \\
- \\
327 \\
377 \\
429 \\
467 \\
499 \\
514 \\
592\end{array}$ \\
\hline
\end{tabular}

ионов, расположенных в центросимметричных позициях, в частности, в позиции $C_{3 i}$ в структуре биксбиита, и люминесценция от таких центров не должна наблюдаться. Присутствие второго оптического центра обусловлено локальным разупорядочением [25] в позиции с симметрией $C_{3 i}$ при вхождении иона $\mathrm{Tm}^{3+}$ в данную матрицу, в результате чего симметрия понижается и позиция становится нецентросимметричной. Подобная картина наблюдалась в кристалле иттриевого скандата, легированного $\mathrm{Nd}^{3+}[18]$. Следовательно, мы можем сделать вывод, что характер вхождения редкоземельной примеси

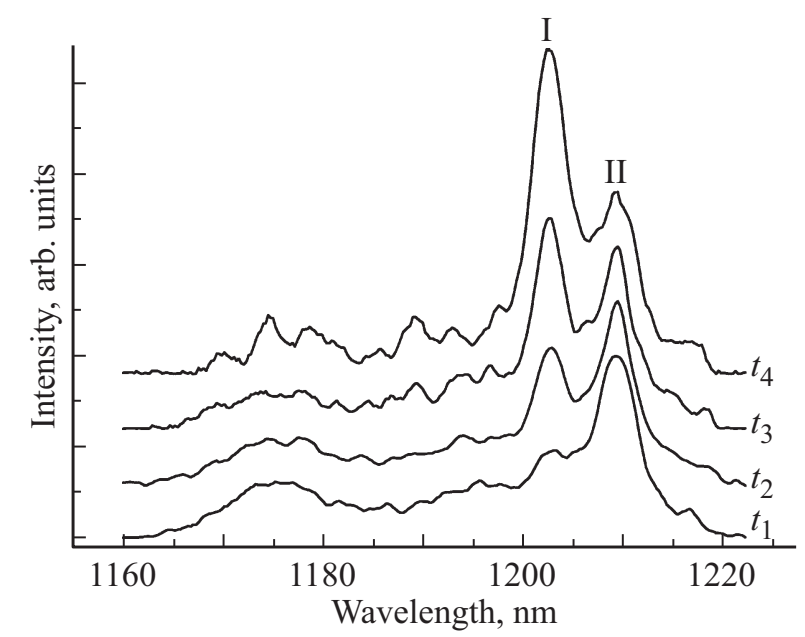

Рис. 4. Спектр возбуждения люминесценции $\mathrm{Tm}^{3+}: \mathrm{YScO}_{3}$ (временные задержки $t_{1}=0.6 \mathrm{~ms}, t_{2}=6 \mathrm{~ms}, t_{3}=10 \mathrm{~ms}$, $\left.t_{4}=12 \mathrm{~ms}\right)$. в кристаллическую структуру данного соединения не зависит от величины радиуса примесного иона.

\section{4. Заключение}

Кристаллическое волокно $\mathrm{Tm}^{3+}: \mathrm{YScO}_{3}$ со структурным типом биксбиита получено методом минипьедестала с лазерным нагревом. Рентгенодифракционные данные указывают на гомогенность образца и высокую степень кристалличности. Результаты анализа спектров комбинационного рассеяния света свидетельствуют о статистическом распределении основных катионов $\mathrm{Y}^{3+}$, $\mathrm{Sc}^{3+}$ между двумя катионными позициями с симметрией $C_{3 i}$ (центросимметричная) и $C_{2}$ (нецентросимметричная) в структуре биксбиита. Методами селективной лазерной спектроскопии выявлено два типа оптических центров ионов $\mathrm{Tm}^{3+}$, находящихся в нецентросимметричных позициях. Природа возникновения второго оптического центра связана с частичным локальным разупорядочением в позиции с симметрией $C_{3 i}$ при вхождении примесного иона $\mathrm{Tm}^{3+}$ в кристаллическую структуру. Сравнение двух кристаллов $\mathrm{YScO}_{3}$, легированных $\mathrm{Nd}^{3+}$ и $\mathrm{Tm}^{3+}$, показало, что спектральное поведение редкоземельных примесей в кристалле не зависит от величины их ионного радиуса.

\section{Финансирование работы}

Работа выполнена при поддержке гранта РНФ № 2222-00968. 


\section{Благодарности}

Рентгенодифракционные измерения проводились на оборудовании ЦКП ИОФ РАН.

\section{Конфликт интересов}

Авторы заявляют об отсутствии конфликта интересов.

\section{Список литературы}

[1] P.H. Klein, W.J. Croft. J. Appl. Phys. 38, 4, 1603 (1967).

[2] Y. Nigara. Jpn J. Appl. Phys. 7, 4, 404 (1968).

[3] K. Petermann. In: Handbook of Solid-State Lasers. Elsevier (2013). P. 3-27.

[4] L. Fornasiero, E. Mix, V. Peters, K. Petermann, G. Huber. Cryst. Res. Technol.: J. Exp. Industrial Cryst. 34, 2, 255 (1999).

[5] Z. Zhou, X. Guan, X. Huang, B. Xu, H. Xu, Z. Cai, X. Xu, P. Liu, D. Li, J. Zhang, J. Xu. Opt. Lett. 42, 19, 3781 (2017).

[6] W. Liu, D. Lu, R. Guo, K. Wu, S. Pan, Y. Hang, D. Sun, H. Yu, H. Zhang. J. Wang. Cryst. Growth Des. 20, 7, 4678 (2020).

[7] S. Li, L. Zhang, X. Tan, W. Deng, M. He, G. Chen, M. Xu, Y. Yang, S. Zhang, P. Zhang, Z. Chen, Y. Hang. Opt.Mater. 96, 109277 (2019).

[8] M.M. Antoinette, S. Israel, A.J. Amali, J.L. Berchmans, B.S. Kumar, G. Manoj, U.M. Usmaniya. Nano-Struct. NanoObjects 13, 51 (2018).

[9] K.R. Devi, S.D. Singh, T.D. Singh. Indian J. Phys. 92, 6, 725 (2018).

[10] E.W. Barrera, C. Cascales, M.C. Pujol, K.H. Park, S.B. Choi, F. Rotermund, J.J. Carvajal, X. Mateos, M. Aguiló, F. Díaz. Phys. Proc. 8, 142 (2010).

[11] A. Kumar, S. Tiwari, K. Kumar, V. Rai. Spectrochim. A 167, 134 (2016)

[12] K.P. Tseng, Q. Yang, S.J. McCormack, W.M. Kriven. J. Am. Ceram. Soc. 103, 1, 569 (2020).

[13] K.E. El-Kelany, C. Ravoux, J. Desmarais, P. Cortona, Y. Pan, J. Tse, A. Erba. Phys. Rev. B 97, 24, 245118 (2018).

[14] S. Balabanov, S. Filofeev, M. Ivanov, E. Kalinina, D. Kuznetsov, D. Permin, E. Rostokina. Heliyon 5, 4, e01519 (2019).

[15] F. Hanic, M. Hartmanová, G. Knab, A. Urusovskaya, K.S. Bagdasarov. Acta Crystallographica B 40, 2, 76 (1984).

[16] S. Geller, P. Romo, J. Remeika. Z. Kristallograph. Cryst. Mater. 124, 1-6, 136 (1967).

[17] J. Clark, P. Richter, L. Du Toit. J. Solid State Chem. 23, 1-2, 129 (1978).

[18] O. Alimov, E. Dobretsova, D. Guryev, V. Kashin, G. Kiriukhina, S. Kutovoi, S. Rusanov, S. Simonov, V. Tsvetkov, V. Vlasov, V. Voronov, O. Yakubovich. Cryst. Growth Des. 20, 7, 4593 (2020).

[19] G.A. Bufetova, V.V. Kashin, D.A. Nikolaev, S.Y. Rusanov, V.F. Seregin, V.B. Tsvetkov, I.A. Shcherbakov, A.A. Yakovlev. Quantum Electron. 36, 7, 616 (2006).

[20] R.S. Feigelson. J. Cryst. Growth 79, 1-3, 669 (1986).

[21] W.B. White, V.G. Keramidas. Spectrochim. Acta A 28, 3, 501 (1972).

[22] D.L. Rousseau, R.P. Bauman, S. Porto. J. Raman Spectroscopy 10, 1, 253 (1981).

[23] M.V. Abrashev, N.D. Todorov, J. Geshev. J. Appl. Phys. 116, 10, 103508 (2014).
[24] А.А. Каминский. Лазерные кристаллы. Наука, М.(1975).

[25] О.К. Алимов, Т.Т. Басиев, С.Б. Миров. Тр. ИОФАН, 9, 6 (1987).

Редактор К.В. Емцев 